\title{
Prevalencia de enfermedad por reflujo gastroesofágico por pH-metría en recién nacidos prematuros con síntomas sugestivos
}

\section{Prevalence of gastroesophageal reflux disease found by $\mathrm{pH}$ measurements in preterm infants with suggestive symptoms}

Carlos Alberto Velasco Benítez, ${ }^{1 *}$ Ernesto León Vallejo Mondragón, ${ }^{2}$ Mauricio Alberto Arévalo Sanabria. ${ }^{3}$

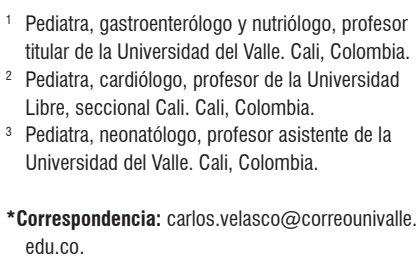
titular de la Universidad del Valle. Cali, Colombia.

2 Pediatra, cardiólogo, profesor de la Universidad Libre, seccional Cali. Cali, Colombia.

3 Pediatra, neonatólogo, profesor asistente de la Universidad del Valle. Cali, Colombia.

*Correspondencia: carlos.velasco@correounivalle. edu.co.

Fecha recibido: $12 / 10 / 18$

Fecha aceptado: 04/02/19

\begin{abstract}
Resumen
Introducción: el reflujo gastroesofágico (RGE), fenómeno fisiológico común en recién nacidos pretérminos (RNPreT), se diagnostica frecuentemente en neonatos, convirtiéndose en un importante fenómeno clínico en pretérminos. Objetivo: determinar la prevalencia y los síntomas de enfermedad por RGE (ERGE) por pHmetría intraesofágica ambulatoria de 24 horas $(\mathrm{pHm})$ en pretérminos. Metodología: estudio de prevalencia en pretérminos de una unidad de cuidados intensivos neonatales de Cali, Colombia a quienes, por sospecha clínica de ERGE, se les solicitó una pHm, teniendo en cuenta variables sociodemográficas y clínicas. La estadística incluyó un análisis univariado por medio de medidas de tendencia central y análisis bivariado con la prueba de chi cuadrado $\left(X^{2}\right)$ y la t de student, siendo significativa una $p<0,05$. Resultados: se analizaron 20 RNPreT de 36,5 \pm 27,6 días de edad postnatal; de 31,6 \pm 3,8 semanas de edad gestacional; 12 masculinos. $11 \mathrm{RNPreT}(55,0 \%)$ presentaron una pHm anormal. La presencia de residuos y cardiopatía estuvieron asociados con pHm anormal. Conclusión: La ERGE por pHm en los RNPreT estudiados tiene una prevalencia relativamente alta en comparación con la literatura mundial, sin encontrar una franca asociación con los síntomas analizados, a excepción de cardiopatía y residuos gástricos.
\end{abstract}

\section{Palabras clave}

Recién nacido, prevalencia, reflujo gastroesofágico, monitorización esofágica.

\begin{abstract}
Introduction: Gastroesophageal reflux is a common physiological phenomenon in preterm infants and is frequently diagnosed in neonates for whom it is an important clinical phenomenon. Objective: To determine the prevalence and symptoms of gastroesophageal reflux disease (GERD) by 24-hour ambulatory esophageal $\mathrm{pH}$ monitoring of preterm neonates. Methodology: This is a study of the prevalence of GERD among patients in the Neonatal Intensive Care Unit of Cali, Colombia. Esophageal pH of infants was monitored when GERD was suspected. In addition, sociodemographic and clinical variables were recorded and taken into account. Univariate analysis by means of measures of central tendency and bivariate analysis were preformed using the chi-squared test and Student's T test with $p<0.05$ established as significant. Results: Twenty preterm newborns whose ages from birth ranged from 27.6 days to 36.5 days and whose gestational ages ranged from 3.8 weeks to 31.6 weeks were included. Twelve were male, and eleven $(55.0 \%)$ had abnormal pH. Gastric waste and heart disease were associated with abnormal pH. Conclusion: The prevalence of GERD found through $\mathrm{pH}$ monitoring was relatively high in this group of infants compared findings in the world literature although no clear associations were found between the symptoms analyzed and other factors except for heart disease and gastric waste.
\end{abstract}

\section{Keywords}

Newborn, prevalence, gastroesophageal reflux, esophageal monitoring. 


\section{INTRODUCCIÓN}

El reflujo gastroesofágico (RGE), definido como la devolución con o sin regurgitación o vómito del contenido gástrico al esófago, debe diferenciarse de la enfermedad por RGE (ERGE), que incluye una serie de síntomas del RGE que afectan la calidad de vida del niño o complicaciones patológicas tales como falla para crecer, problemas en la alimentación o en el sueño, problemas respiratorios crónicos, esofagitis, hematemesis, apnea y eventos aparentes que amenazan la vida $(1,2)$. El RGE, además de ser un fenómeno fisiológico común en recién nacidos pretérminos (RNPreT) (3), es un diagnóstico frecuente en las unidades de cuidados intensivos neonatales (UCIN), lo que conlleva a estancias hospitalarias prolongadas y elevados costos intrahospitalarios, por lo que se convierte en un importante fenómeno clínico en las UCIN (4).

El RGE en RNPreT es a menudo diagnosticado y manejado con base en las manifestaciones clínicas más que a paraclínicos específicos; y es escasa la evidencia del daño que causa el RGE en los RNPreT y, por ende, cuestionables los medicamentos anti-RGE usados de rutina en este grupo etario (4).

Entre los signos y síntomas inespecíficos, entre otros, atribuibles a RGE están: intolerancia o aversión a los alimentos, pobre ganancia de peso, regurgitación frecuente, apnea, desaturación, bradicardia, arqueo e irritabilidad $(1,3,4)$.

La monitorización del $\mathrm{pH}$ en el esófago inferior clásicamente se ha usado para el diagnóstico de RGE en niños y adultos, por medio de la $\mathrm{pH}$-metría intraesofágica ambulatoria de 24 horas $(\mathrm{pHm})$ y la impedanciometría intraluminal multicanal (MII). La MII, además de medir los movimientos esofágicos a líquidos, sólidos y aire por impedanciometría eléctrica, y si los movimientos son anterógrados o retrógrados, simultáneamente combina los parámetros de una $\mathrm{pHm}$ (5). Los valores normales de referencia a la pHm en RNPreT son los de Ng y colaboradores (6).

El objetivo del presente estudio es determinar la prevalencia y los síntomas de ERGE por pHm en RNPreT del Hospital Universitario del Valle (HUV) Evaristo García de Cali, Colombia.

\section{MATERIAL Y MÉTODOS}

Se realizó un estudio observacional, descriptivo, no experimental, de corte trasversal, comparativo, en RNPreT que fueron hospitalizados en Cuidados Intensivos del Recién Nacido (CIRENA) del HUV Evaristo García de Cali, Colombia, entre enero y junio de 2013, con diagnóstico de prematurez y con patologías típicas de su estado como síndrome de dificultad respiratoria, sepsis y trastornos metabólicos.
Se incluyeron los recién nacidos prematuros con síntomas sugestivos de ERGE como desaturación durante la alimentación, distensión, vómito, apnea inexplicable, residuo, tos, llanto, irritabilidad, eventos aparentes que amenazan la vida y falta de ganancia de peso, no atribuibles a otra patología. Se excluyeron los RNPreT que hubieran recibido medicamentos antirreflujo antes de ser incluidos en la investigación. Antes de iniciar el estudio, a los acudientes del RNPreT se les describió la naturaleza y el propósito de la investigación, teniendo la oportunidad de solicitar mayor información y discutir cualquier consulta que tuvieran. Todos los neonatos estaban alimentados enteralmente al momento del estudio y algunos de ellos con alimentación nasogástrica in situ, además se encontraban en su posición de sueño normal, fuera en supino o prono.

A cada RNPreT se le realizó una pH-metría intraesofágica ambulatoria ( $\mathrm{pHm}$ ), mínimo por 20 horas continuas; previo ayuno de 4 horas: se les colocó un catéter pediátrico desechable de 1 canal con punta de antimonio, unido al pH-metro Mark III de Synetics Medical, que se calibró antes del estudio con soluciones buffer a un $\mathrm{pH}$ de 4,0 y de 7,0 . La longitud a introducir por una de las narinas del RN de cada catéter se calculó con base en la fórmula de Strobel y colaboradores (7) (talla x $0,252+5 \times 0,86)$, y corroborada por una radiografía de tórax portátil que evidenciara que la punta del catéter quedara ubicada entre la sexta y la octava vértebra torácica, luego de lo cual fue asegurada con esparadrapo para evitar desplazamientos del mismo durante el tiempo de duración de la pHm. Se llevó a cabo un registro horario de síntomas y signos presentes durante la realización del procedimiento.

$\mathrm{El}$ análisis de los trazados de la pH-metría se realizó utilizando el software de Synetics Medical, e interpretado según los valores normales estándar descritos por $\mathrm{Ng}$ y colaboradores (6), teniendo en cuenta el índice de reflujo, el número de episodios ácidos, el número de episodios ácidos mayores de 5 minutos y la duración del episodio ácido más prolongado.

El análisis estadístico realizado con el software Stata 15 incluyó medidas de tendencia central como porcentajes, promedios y desviaciones estándar; y análisis univariado usando la prueba de chi cuadrado $\left(\chi^{2}\right)$ y la $t$ de student, siendo significativa una $p<0,05$.

De acuerdo con la Resolución 8430 de 1993 del Ministerio de Salud de Colombia, según las normas éticas de la investigación con seres humanos, este estudio se clasificó como de riesgo mínimo; además, según la Declaración de Helsinki, se garantizaron los derechos y el bienestar de cada participante. Los padres o tutores firmaron un documento de consentimiento informado antes de incluirse en el estudio, teniendo en cuenta que los datos obtenidos iban a ser utilizados en una investigación, asegurando la confi- 
dencialidad, el manejo profesional de los datos y los resultados derivados del estudio.

\section{RESULTADOS}

\section{Características generales}

Se realizó un estudio observacional, descriptivo, no experimental, de tipo corte trasversal, comparativo, en 20 RNPreT con edad posnacimiento de 36,5 $\pm 27,6$ días, edad gestacional de 31,6 $\pm 3,8$ semanas, peso de 1493,3 $\pm 579,8$ gramos, Ballard de $31,6 \pm 3,7$ semanas, 12 RNpreT de sexo masculino, 18 de raza mestiza y 17 originarios de Cali, Colombia. Las madres tenían edad de 25,0 \pm 7,4 años; 10 sin control prenatal, 10 multíparas, 2 con parto vaginal y 8 con cesárea. Se hospitalizaron por su condición de prematurez y con patologías típicas de su estado como síndrome de dificultad respiratoria, sepsis y trastornos metabólicos.

\section{Valores de la pH-metría intraesofágica ambulatoria}

11 de los 20 RNPreT (55,0 \%) presentaron una pH-metría intraesofágica ambulatoria anormal (Tabla $\mathbf{1}$ ).

Tabla 1. Valores de la pH-metría intraesofágica ambulatoria en 11 RNPreT del Hospital Universitario del Valle Evaristo García de Cali, Colombia

\begin{tabular}{lcc}
\hline \multicolumn{1}{c}{ Variable } & $\begin{array}{c}\text { Promedio } \\
(\mathbf{n}=11)\end{array}$ & $\begin{array}{c}\text { Valores normales } \\
\text { según } \mathbf{~ N g} \text { et al }(6)\end{array}$ \\
\hline Duración del estudio (horas) & 21,4 & \\
Índice de reflujo (\%) & 18,1 & $0,7+1,1$ \\
Número de episodios ácidos & 157 & $7,6+11,2$ \\
Número de episodios ácidos $>5$ & 13,7 & $0,5+1,1$ \\
$\begin{array}{l}\text { minutos } \\
\begin{array}{l}\text { Duración del episodio más largo } \\
\text { (minutos) }\end{array}\end{array}$ & 70 & $4,6+6,1$ \\
\hline
\end{tabular}

\section{Signos y síntomas y pH-metría intraesofágica ambulatoria}

La presencia de residuos y una cardiopatía en los 20 RNPreT estuvieron asociados con los resultados anormales de la pH-metría (Tabla 2).

\section{DISCUSIÓN}

La pH-metría $(\mathrm{pHm})$ o impedanciometría intraluminal multicanal/pH-metría $(\mathrm{MII} / \mathrm{pHm})$ es una práctica relativamente común en la investigación de la enfermedad por reflujo gastroesofágico (ERGE) en recién nacidos pretérminos (RNPreT); siendo solicitada entre un $24,0 \%$ (8) y
Tabla 2. Signos y síntomas asociados y pH-metría intraesofágica ambulatoria en 20 RNPreT del Hospital Universitario del Valle Evaristo García de Cali, Colombia

\begin{tabular}{|c|c|c|c|c|c|c|c|}
\hline & \multicolumn{2}{|c|}{ pH-metría } & \multirow[b]{2}{*}{$p$} & & \multicolumn{2}{|c|}{ pH-metría } & \multirow[b]{2}{*}{$p$} \\
\hline & Normal & Anormal & & & Normal & Anormal & \\
\hline \multicolumn{3}{|c|}{ Cianosis } & \multicolumn{4}{|c|}{ Tos } & \multirow{3}{*}{0,085} \\
\hline Sí & 5 & 4 & \multirow{2}{*}{0,203} & Sí & 6 & 5 & \\
\hline No & 15 & 7 & & No & 14 & 6 & \\
\hline \multicolumn{4}{|c|}{ Apnea } & \multicolumn{4}{|c|}{ Síndrome de dificultad respiratoria } \\
\hline Sí & 11 & 8 & \multirow{2}{*}{0,086} & Sí & 11 & 6 & \multirow{2}{*}{0,996} \\
\hline No & 9 & 3 & & No & 9 & 5 & \\
\hline \multicolumn{3}{|c|}{ Vómito } & \multicolumn{4}{|c|}{ Regurgitación } & \multirow{3}{*}{0,731} \\
\hline Sí & 13 & 6 & \multirow{2}{*}{0,303} & Sí & 8 & 4 & \\
\hline No & 7 & 5 & & No & 12 & 7 & \\
\hline \multicolumn{3}{|c|}{ Residuos gástricos } & \multicolumn{4}{|c|}{ Distensión abdominal } & \multirow{3}{*}{0,85} \\
\hline Sí & 10 & 3 & \multirow{2}{*}{0,024} & Sí & 3 & 1 & \\
\hline No & 10 & 8 & & No & 17 & 10 & \\
\hline \multicolumn{3}{|c|}{ Sialorrea } & \multicolumn{5}{|c|}{ Enterocolitis necrosante } \\
\hline Sí & 1 & 0 & \multirow{2}{*}{0,281} & Sí & 1 & 1 & \multirow{2}{*}{0,38} \\
\hline No & 19 & 11 & & No & 19 & 10 & \\
\hline \multicolumn{3}{|c|}{ Falta de ganancia de peso } & \multicolumn{4}{|c|}{ Irritabilidad } & \multirow{3}{*}{0,38} \\
\hline Sí & 1 & 1 & \multirow{2}{*}{1} & Sí & 1 & 1 & \\
\hline No & 19 & 10 & & No & 19 & 10 & \\
\hline \multicolumn{3}{|c|}{ Cardiopatía } & \multicolumn{4}{|c|}{ Sepsis } & \multirow{3}{*}{0,673} \\
\hline Sí & 3 & 3 & \multirow{2}{*}{0,001} & Sí & 10 & 5 & \\
\hline No & 17 & 8 & & No & 10 & 6 & \\
\hline Uso d & aminofilir & & & Uso de & antibiótic & & \\
\hline Sí & 8 & 5 & & Sí & 18 & 9 & \\
\hline No & 12 & 6 & U,0U5 & No & 2 & 2 & 0,190 \\
\hline
\end{tabular}

$32,0 \%$ (9) en unidades de cuidados neonatales. Algunos autores comentan que, con los estudios del momento, no se puede estimar la validez de las pruebas diagnósticas de ERGE, entre ellas la pH-metría, y que son necesarios ensayos clínicos en los que se valore la utilidad de otras pruebas para la toma de decisiones (10-12). Según las recomendaciones de las actuales Guías Clínicas Prácticas para Reflujo Gastroesofágico Pediátrico de las Sociedades Norteamericana y Europea de Gastroenterología, Hepatología y Nutrición Pediátrica (NASPGHAN/ESPGHAN), cuando no es posible realizar una $\mathrm{MII} / \mathrm{pHm}$, se puede considerar el uso de la pHm, entre otros, cuando se quieren correlacionar los síntomas persistentes con los eventos de reflujo gastroesofágico ácido (2); este estudio utiliza como prueba diagnóstica la pHm para el diagnóstico de la ERGE, paraclínico que detecta más episodios de reflujo que la $\mathrm{MII} / \mathrm{pHm}$, según lo mencionan Rossor y colaboradores (13). 


\section{Prevalencia de ERGE en RNPreT}

Más de la mitad de los RNPreT estudiados en CIRENA del HUV de Cali, Colombia, presentaron una pHm anormal; datos muy similares a los descritos por Di Fiore y colaboradores (14), quienes reportaron un 59,0 \% de ERGE diagnosticada por $\mathrm{pHm}$; y resultados superiores a los identificados por MII/pHm de Sivalingam y colaboradores (15) del 30,0 \%, de Rossor y colaboradores (13) del 21,0 \% y de Funderburk y colaboradores (16), del 10,0\%.

La variabilidad en estas prevalencias podría ser resultado de las diferentes técnicas e interpretación de los resultados obtenidos para hacer el diagnóstico de ERGE, así como de otras características sociodemográficas, de la edad postnatal y la edad gestacional de los RNPreT estudiados en cada una de estas investigaciones; lo que implica una mayor estandarización y posterior análisis. Así mismo, es posible que los resultados pueden estar influenciados por el número total de los RNPreT estudiados, que al ser una muestra pequeña puede dar una prevalencia mayor en el grupo estudiado, ya que la población analizada fue la que tenía sospecha de ERGE.

\section{Signos y síntomas asociados a ERGE en RNPreT}

Son pocos los estudios que mencionan la asociación entre los síntomas y la presencia de ERGE, es por ello que se han utilizado ciertos índices en la interpretación de la MII/ $\mathrm{pHm}$, que puedan realizar una mayor aproximación diagnóstica de ERGE en neonatos; sin embargo, Barriga Rivera y colaboradores (17), en neonatos con síntomas cardiorespiratorios, no lograron demostrar que el índice de síntomas (IS), el índice de sensibilidad de síntomas (ISS) y la probabilidad de asociación de síntomas (PAS) sean de utilidad para este fin. La relación entre ERGE y eventos cardiorrespiratorios en neonatos es controversial (18). En nuestros resultados, los niños con cardiopatía sin síntomas respiratorios tuvieron hallazgos anormales a la $\mathrm{pHm}$ en comparación con los niños sin cardiopatía $(p<0,05)$; datos que podrían ser correspondientes a los relatados por Qureshi y colaboradores (19), quienes solamente encuentran un 6,1\% de los síntomas cardiorespiratorios durante el sueño asociados a eventos con diagnóstico de ERGE por $\mathrm{MII} / \mathrm{pHm}$ y polisomnografía concurrente.

En este estudio no hubo ningún RNPreT con eventos aparentes que amenazaran la vida, pero Macchini y colaboradores (20) informaron un $80,0 \%$ de estos síntomas en niños con diagnóstico de ERGE por $\mathrm{pHm}$. A pesar de que la ERGE y la apnea son frecuentes en los RNPreT, al igual que Rossor y colaboradores (21), nosotros no encontramos ninguna asociación entre la ERGE y la presencia de apnea.
En relación con la alimentación de los RNPreT de nuestro estudio, la presencia del residuo para nosotros fue estadísticamente significativa para presentar una $\mathrm{pHm}$ anormal; sin embargo, se requiere de una mayor observación en la alimentación de estos neonatos para identificar otros posibles confusores; y a pesar de que nosotros no tuvimos en cuenta si al RNPreT se le estimulaba su nutrición no nutritiva con el uso del chupo, Corvaglia y colaboradores (22), en un estudio de corte transversal en RNPreT, sugirieron que el chupo puede ser razonablemente utilizado en estos neonatos, pues su uso no tuvo efectos en el RGE ácido y no ácido evaluado por $\mathrm{MII} / \mathrm{pHm}$.

Seguimos en este sentido, teniendo una limitada causalidad entre los eventos de reflujo y los signos anormales en RNPreT. Entonces, ante la sospecha clínica de ERGE en RNPreT, se deberían realizar procedimientos diagnósticos como la MII/pHm (23); sin embargo, Salvatore y colaboradores (24) encontraron una pobre correlación entre el informe parental de los síntomas de ERGE, el resultado del estudio de la pHm y la evidencia endoscópica de esofagitis por ERGE. En relación con la indicación de la $\mathrm{MII} / \mathrm{pHm}$, según lo investigado por De Rose y colaboradores (25) en RNPreT, no solo juega un papel diagnóstico, sino también ofrece un valor pronóstico en términos de la duración del tratamiento farmacológico. En este sentido terapéutico, Loots y colaboradores (26) lograron demostrar que el manejo con inhibidores de la bomba de protones (IBP; omeprazol a $1 \mathrm{mg} / \mathrm{kg} /$ día o esomeprazol a $0,5 \mathrm{mg} / \mathrm{kg} /$ día por 2 semanas) mejora la integridad de la mucosa esofágica en lactantes entre los 0 y 6 meses de edad con diagnóstico de ERGE por $\mathrm{MII} / \mathrm{pHm}$.

Entre las debilidades del estudio están la asociada con el tipo de alimentación y el residuo gástrico de los RNPreT alimentados por sonda nasogástrica in situ en cuanto a la manera de cuantificación, el volumen, los niños con o sin sonda nasogástrica, con presencia o ausencia de residuo gástrico y en posición prona o supina; debido a que la presencia de la sonda nasogástrica puede ser un factor confusor y además un factor de riesgo que aumenta los episodios de ERGE; y que en posición prona, los episodios de ERGE son menores.

En conclusión, la ERGE por pHm en los RNPreT estudiados tiene una prevalencia relativamente alta en comparación con la literatura mundial, sin encontrar una franca asociación con los síntomas analizados, a excepción de la cardiopatía y los residuos gástricos, que finalmente no se lograron analizar por desconocimiento en términos de cuantificación, volumen, presencia de sonda nasogástrica y posición; lo que enfatiza la necesidad de realizar mayores estudios para determinar estas posibles asociaciones $y$, de este modo, un apropiado manejo de la ERGE. 


\section{REFERENCIAS}

1. Czinn SJ, Blanchard S. Gastroesophageal reflux disease in neonates and infants: When and how to treat. Pediatr Drugs. 2013;15:19-27. doi: 10.1007/s40272-012-0004-2.

2. Rosen R, VandenplasY,SingendonkM,CabanaM,DiLorenzo C, Gottrand F, et al. Pediatric Gastroesophageal Reflux Clinical Practice Guidelines: Joint Recommendations of the North American Society for Pediatric Gastroenterology, Hepatology, and Nutrition and the European Society for Pediatric Gastroenterology, Hepatology, and Nutrition. J Pediatr Gastroenterol Nutr. 2018;66(3):516-554. doi: 10.1097/MPG.0000000000001889.

3. Kültürsay N. Gastroesophageal reflux (GER) in preterms: Current dilemmas and unresolved problems in diagnosis and treatment. Turk J Pediatr. 2012;54:561-9.

4. Eichenwald EC. Diagnosis and Management of Gastroesophageal Reflux in Preterm Infants. Pediatrics. 2018;142(1). pii: e20181061. doi: 10.1542/peds.2018-1061.

5. Velasco Benítez CA. GERD in children: An update | Actualización sobre enfermedad por reflujo gastroesofágico en niños. Rev Colomb Gastroenterol. 2014;29:55-62.

6. Ng SCY, Quak SH. Gastroesophageal reflux in preterm infants: Norms for extended distal esophageal $\mathrm{pH}$ monitoring. J Pediatr Gastroenterol Nutr. 1998;27:411-4. doi: 10.1097/00005176-199810000-00009.

7. Strobel CT, Byrne WJ, Ament ME, Euler AR. Correlation of esophageallengths in children with height: Application to the Tuttle test without prior esophageal manometry. J Pediatr. 1979;94:81-4. doi: 10.1016/S0022-3476(79)80361-3.

8. Rossor T, Andradi G, Bhat R, Greenough A. Investigation and management of gastro-oesophageal reflux in United Kingdom neonatal intensive care units. Acta Paediatr. 2018;107:48-51. doi: 10.1111/apa.14073.

9. Dhillon AS, Ewer AK. Diagnosis and management of gastrooesophageal reflux in preterm infants in neonatal intensive care units. Acta Paediatr Int J Paediatr. 2004;93:88-93. doi: 10.1080/08035250310007934.

10. Díaz JJ. ¿Podemos diagnosticar adecuadamente el reflujo gastroesofágico en niños? Evid Pediatr. 2013;9:59.

11. Ochoa C, de Llano A. La validez de las pruebas diagnósticas de la enfermedad por reflujo gastroesofágico en la infancia es dudosa. Evid Pediatr. 2013;9:63.

12. van derPolRJ, Smits MJ,Venmans L, BoluytN,Benninga MA, Tabbers MM. Diagnostic accuracy of tests in pediatric gastroesophageal reflux disease. J Pediatr. 2013;162(5):983-7. e1-4. doi: 10.1016/j.jpeds.2012.10.041.

13. Rossor T, Lingam I, Douiri A, Bhat R, Greenough A. Detection of gastro-oesophageal reflux in the neonatal unit. Acta Paediatr Int J Paediatr. 2018;107:1535-40. doi: 10.1111/apa.14315.

14. Di Fiore J, Arko M, Churbock K, Hibbs A, Martin R. Technical limitations in detection of gastroesophageal reflux (GER) in neonates. J Pediatr Gastroenterol Nutr. 2009;49:177-82. doi: 10.1097/MPG.0b013e318195d7b3.

15. Sivalingam M, Sitaram S, Hasenstab K, Wei L, Woodley F, Jadcheria S. Effects of esophageal acidification on trou- blesome symptoms: an approach to characterize true acid GERD in dysphagic neonates. Dysphagia. 2017;32:509-19. doi: $10.1007 /$ s00455-017-9792-4.

16. Funderburk A, Nawab U, Abraham S, DiPalma J, Epstein M, Aldridge $\mathrm{H}$, et al. Temporal association between reflux-like behaviors and gastroesophageal reflux in preterm and term infants. J Pediatr Gastroenterol Nutr. 2016;62:556-61. doi: 10.1097/MPG.0000000000000968.

17. Barriga-Rivera A, Moya MJ, Lopez-Alonso M. El índice de síntomas binomial para la evaluación de la asociación temporal entre síntomas cardiorrespiratorios y reflujo gastroesofágico en neonatos. An Pediatr. 2016;85:232-9. doi: 10.1016/j.anpedi.2015.09.024.

18. Lopez-Alonso M, Moya MJ, Cabo JA, Ribas J, del Carmen Macias M, Silny J, et al. Twenty-Four-Hour Esophageal Impedance-pH Monitoring in Healthy Preterm Neonates: Rate and Characteristics of Acid, Weakly Acidic, and Weakly Alkaline Gastroesophageal Reflux. Pediatrics. 2006;118:e299-308. doi: 10.1542/peds.2005-3140.

19. Qureshi A, Malkar M, Splaingard M, Khuhro A, Jadcheria $S$. The role of sleep in the modulation of gastroesophageal reflux and symptoms in NICU neonates. Pediatr Neurol. 2015;53:226-32. doi: 10.1016/j.pediatrneurol.2015.05.012.

20. Macchini F, Morandi A, Cognizzoli P, Farris G, Gentilino V, Zanini A, et al. Acid Gastroesophageal Reflux Disease and Apparent Life-Threatening Events: Simultaneous pHmetry and Cardiorespiratory Monitoring. Pediatr Neonatol. 2017;58:43-7. doi: 10.1016/j.pedneo.2015.12.005.

21. Rossor T, Andradi G, Ali K, Bhat R, Greenough A. GastroOesophageal Reflux and Apnoea: Is There a Temporal Relationship? Neonatology. 2018;113:206-11. doi: 10.1159/000485173.

22. Corvaglia L, Martini S, Corrado MF, Mariani E, Legnani E, Bosi I, et al. Does the Use of Paci fi er Affect GastroEsophageal Re $\mathrm{fl}$ ux in Preterm Infants? J Pediatr. 2016;172:205-8. doi: 10.1016/j.jpeds.2016.01.022.

23. Corvaglia L, Mariani E, Aceti A, Capretti MG, Ancora G, Faldella G. Combined oesophageal impedance-pH monitoring in preterm newborn: Comparison of two options for layout analysis. Neurogastroenterol Motil. 2009;21:102732. doi: 10.1111/j.1365-2982.2009.01301.x.

24. Salvatore S, Hauser B, Vandemaele K, Novario R, Vandenplas Y. Gastroesophageal Reflux Disease in Infants: How Much is Predictable with Questionnaires, $\mathrm{pH}$-metry, Endoscopy and Histology? J Pediatr Gastroenterol Nutr. 2005;40:210-5. doi: 10.1097/00005176-200502000-00024.

25. De Rose DU, Cresi F, Romano V, Barone G, Fundarò C, Filoni S, et al. Can MII-pH values predict the duration of treatment for GERD in preterm infants? Early Hum Dev. 2014;90:501-5. doi: 10.1016/j.earlhumdev.2014.07.003.

26. Loots CM, Wijnakker R, van Wijk MP, Davidson G, Benninga MA, Omari TI. Esophageal impedance baselines in infants before and after placebo and proton pump inhibitor therapy. Neurogastroenterol Motil. 2012;24(8):758-62, e351-2. doi: 10.1111/j.1365-2982.2012.01922.x. 